\title{
ON A NONVANISHING OF PLURIGENUS OF A THREEFOLD OF GENERAL TYPE
}

\author{
DONG-KHAN SHIN
}

\begin{abstract}
Even though there is a formula for $h^{0}\left(X, \mathcal{O}_{X}\left(n K_{X}\right)\right)$ for a canonical threefold $X$, it is not easy to compute $h^{0}\left(X, \mathcal{O}_{X}\left(n K_{X}\right)\right)$ because the formula has a term due to singularities. In this paper, we find a way to control the term due to singularities. We show nonvanishing of plurigenus for the case when the index $r$ in the singularity type $\frac{1}{r}(1,-1, b)$ is sufficiently large.
\end{abstract}

Throughout this paper $X$ is assumed to be a projective threefold with only canonical singularities and an ample canonical divisor $K_{X}$ over the complex number field $\mathbb{C}$, i.e., a canonical threefold.

It is well known that $H^{0}\left(X, \mathcal{O}_{X}\left(m K_{X}\right)\right)$ does not vanish and generates a birational map for a sufficiently large $m$. When $X$ is a surface of general type, $H^{0}\left(X, \mathcal{O}_{X}\left(m K_{X}\right)\right)$ does not vanish for $m \geq 2$ and $H^{0}\left(X, \mathcal{O}_{X}\left(m K_{X}\right)\right)$ generates a birational map for $m \geq 5$.

In a case of a threefold of general type, M. Reid and A. R. Fletcher described the formula for $\chi\left(\mathcal{O}_{X}\left(n K_{X}\right)\right.$ ) (see [1]). Combining the formula for $\chi\left(\mathcal{O}_{X}\left(n K_{X}\right)\right)$ with a vanishing theorem, we may have a way to compute $h^{0}\left(X, \mathcal{O}_{X}\left(n K_{X}\right)\right)$. But it is not easy to compute $h^{0}\left(X, \mathcal{O}_{X}\left(n K_{X}\right)\right)$ because the formula has a term due to singularities in a case of a threefold. However, A. R. Fletcher showed that $h^{0}\left(X, \mathcal{O}_{X}\left(12 K_{X}\right)\right) \geq 1$ and $h^{0}\left(X, \mathcal{O}_{X}\left(24 K_{X}\right)\right) \geq 2$ when $\chi\left(\mathcal{O}_{X}\right)=1$ in [1]. In [3], Shin showed that $h^{0}\left(X, \mathcal{O}_{X}\left(n K_{X}\right)\right) \geq 2$ for $n \geq 10$ when $\chi\left(\mathcal{O}_{X}\right)=1$ and that $h^{0}\left(X, \mathcal{O}_{X}\left(n K_{X}\right)\right) \geq 2$ for $n \geq 26$ when $\chi\left(\mathcal{O}_{X}\right)=2$.

The formula for $\chi\left(\mathcal{O}_{X}\left(n K_{X}\right)\right)$ is given as follows:

$$
\chi\left(\mathcal{O}_{X}\left(n K_{X}\right)\right)=\frac{n(n-1)(2 n-1)}{12} K_{X}^{3}+(1-2 n) \chi\left(\mathcal{O}_{X}\right)+\sum_{Q} l(Q, n),
$$

where the summation is over a basket of singularities. Although singularities in a basket are not necessarily singularities in $X$, the singularities in $X$ make

Received May 27, 2009; Revised July 7, 2009.

2000 Mathematics Subject Classification. 14E05, 14J99.

Key words and phrases. pluricanonical system, plurigenus, threefold of general type.

This paper was supported by Konkuk University 2008. 
the contribution as if they were in a basket. For detailed explanations about a basket of singularities (see [2], [1]). The exact formula for $l(Q, n)$ is as follows:

$$
l(Q, n)=\sum_{i=1}^{n-1} \frac{\overline{i b}(r-\overline{i b})}{2 r}, \quad \overline{i b}=i b-\left\llcorner\frac{i b}{r}\right\lrcorner r
$$

where $Q$ is a singularity of type $\frac{1}{r}(1,-1, b), r$ and $b$ are relatively prime.

For the sake of simplicity, denote $\sum_{Q} l(Q, n)$ by $l(n)$.

Let's denote the singularity type $\frac{1}{r}(1,-1, b)$ by $b / r$ unless there is some confusion. Moreover, we identify the singularity type $b / r$ with the number $b / r$ in the interval $(0,1)$. By identifying the type $b / r$ with the number $b / r$ in $(0,1)$, our situation in the computation of $l(n)$ can be described more effectively and clearly.

The following proposition is a standard application of the Kawamata-Viehweg Vanishing Theorem.

Proposition 1. For all $n \geq 2$,

$$
p_{n}: \stackrel{\text { def }}{=} h^{0}\left(X, \mathcal{O}_{X}\left(n K_{X}\right)\right)=\frac{n(n-1)(2 n-1)}{12} K_{X}^{3}+(1-2 n) \chi\left(\mathcal{O}_{X}\right)+l(n) .
$$

When $\chi\left(\mathcal{O}_{X}\right) \leq 0$, for example when $X$ is smooth minimal, $p_{n} \geq 2$ for $n \geq 4$. In a general case, even though there is a formula for $p_{n}$, it is not easy to compute $p_{n}$ without full information about the basket of singularities.

In [3], to check the nonvanishing of $p_{4}$, it was needed to find all the types of singularities such that $E q(b, r) \leq 35 \chi\left(\mathcal{O}_{X}\right)$, where

$$
E q(b, r): \stackrel{\text { def }}{=}-\frac{\overline{3 b}(r-\overline{3 b})}{2 r}-\frac{\overline{2 b}(r-\overline{2 b})}{2 r}+13 \frac{\bar{b}(r-\bar{b})}{2 r} .
$$

But, values of $E q(b, r)$ are given as follows:

$$
E q(b, r)= \begin{cases}4 b & \text { if } b / r \leq \frac{1}{3} \\ r+b & \text { if not. }\end{cases}
$$

It means that there are infinitely many points in the interval $(0,1 / 3]$ which have the same value of $E q(b, r)$. So, it is impossible to find all the types we want. Thus, we need to find a way to give a restriction on $r$. In [3], a trick was developed in this direction. In this paper, we present another one: in Theorem 1, we give an upper bound on $r$ imposed by the inequality $p_{n} \leq 1$. This kind of result was treated originally in [1], but our result provides an improvement.

The following lemmas are needed to compute the plurigenus of $X$.

Lemma 1. Let $Q$ be a singularity of type $b / r$. For $1 \leq i, j \leq r-1$,

$$
\overline{i b}(r-\overline{i b})=\overline{j b}(r-\overline{j b}) \Longleftrightarrow i=j \text { or } i+j=r .
$$


Proof. The graph of $x(r-x)$ shows that $\overline{i b}(r-\overline{i b})=\overline{j b}(r-\overline{j b}) \Longleftrightarrow \overline{i b}=\overline{j b}$ or $\overline{i b}+\overline{j b}=r$. Clearly, $\overline{i b}=\overline{j b}$ or $\overline{i b}+\overline{j b}=r \Longleftrightarrow i=j$ or $i+j=r$.

Lemma 2. For a singularity of type $1 / r$ and an integer $n>1$, we have

$$
l(1 / r, n)=q \frac{r^{2}-1}{12}+\frac{s(s+1)}{4}-\frac{s(s+1)(2 s+1)}{12 r},
$$

where $n-1=q r+s(0 \leq s<r)$.

Proof. The formula for $l(1 / r, n)$ is given above. Let's compute $l(1 / r, n)$.

$$
\begin{aligned}
l(1 / r, n) & =\sum_{i=1}^{n-1} \frac{\bar{i}(r-\bar{i})}{2 r} \\
& =\sum_{i=1}^{q r} \frac{\bar{i}(r-\bar{i})}{2 r}+\sum_{i=q r+1}^{q r+s} \frac{\bar{i}(r-\bar{i})}{2 r} \\
& =q \sum_{i=1}^{r-1} \frac{i(r-i)}{2 r}+\sum_{i=1}^{s} \frac{i(r-i)}{2 r} \\
& =q\left(\frac{(r-1) r}{4}-\frac{(r-1)(2 r-1)}{12}\right)+\frac{s(s+1)}{4}-\frac{s(s+1)(2 s+1)}{12 r} \\
& =q \frac{r^{2}-1}{12}+\frac{s(s+1)}{4}-\frac{s(s+1)(2 s+1)}{12 r} .
\end{aligned}
$$

Proposition 2. For a singularity of type $b / r$ and an integer $n>1$, let $n-1=$ $q r+s(0 \leq s<r)$. Then

$$
l(b / r, n) \begin{cases}\geq l(1 / r, n) & \text { when } s \leq\left\llcorner\frac{r}{2}\right\lrcorner, \\ \leq l(1 / r, n) & \text { when } s \geq\left\llcorner\frac{r}{2}\right\lrcorner .\end{cases}
$$

Proof. The formula for $l(b / r, n)$ is given as follows:

$$
\begin{aligned}
l(b / r, n) & =\sum_{i=1}^{n-1} \frac{\overline{i b}(r-\overline{i b})}{2 r} \\
& =\sum_{i=1}^{q r} \frac{\overline{i b}(r-\overline{i b})}{2 r}+\sum_{i=q r+1}^{q r+s} \frac{\overline{i b}(r-\overline{i b})}{2 r} \\
& =q \sum_{i=1}^{r-1} \frac{i(r-i)}{2 r}+\sum_{i=1}^{s} \frac{\overline{i b}(r-\overline{i b})}{2 r} .
\end{aligned}
$$

The difference between $l(b / r, n)$ and $l(1 / r, n)$ is caused by the last term

$$
\sum_{i=1}^{s} \frac{\overline{i b}(r-\overline{i b})}{2 r}
$$


in the formula. So, let's consider only the last term.

When $s \leq\left\llcorner\frac{r}{2}\right\lrcorner$, Lemma 1 shows that the smallest $s$ values of $x(r-x)$ appear in the last term of $l(1 / r, n)$.

When $s \geq\left\llcorner\frac{r}{2}\right\lrcorner$, the sum from $i=1$ to $\left\llcorner\frac{r}{2}\right\lrcorner$ is same for both $l(b / r, n)$ and $l(1 / r, n)$. In the sum from $i=\left\llcorner\frac{r}{2}\right\lrcorner+1$ to $s$, the largest $s-\left\llcorner\frac{r}{2}\right\lrcorner$ values of $x(r-x)$ appear in the last term of $l(1 / r, n)$. Thus, our claim is proved.

When $\chi\left(\mathcal{O}_{X}\right) \leq 0, p_{n}$ can be computed easily, so we just consider only the case $\chi\left(\mathcal{O}_{X}\right)>0$ in Theorem 1 .

Theorem 1. Let $X$ be a canonical threefold with $\chi\left(\mathcal{O}_{X}\right)>0$. Let two integers $r, n$ satisfy the following:

1) $n \geq 8 \chi\left(\mathcal{O}_{X}\right)+2$,

2) $r \geq \max \left\{2 n-2, \frac{2 n^{2}-3 n+1}{3 n-24 \chi\left(\mathcal{O}_{X}\right)-3}\right\}$.

If the basket of singularities contains a singularity of type $b / r$, then $p_{n} \geq 2$.

Proof. Let's compute $l(n)$ :

$$
\begin{aligned}
l(n) & \geq l(b / r, n) \\
& \geq l(1 / r, n) \text { by Proposition } 2 \text { since } n-1 \leq\left\llcorner\frac{r}{2}\right\lrcorner \\
& =\frac{n(n-1)}{4}-\frac{n(n-1)(2 n-1)}{12 r} \text { by Lemma } 2 .
\end{aligned}
$$

Therefore,

$$
\begin{aligned}
l(n)-(2 n-1) \chi\left(\mathcal{O}_{X}\right) & \geq \frac{n(n-1)}{4}-\frac{n(n-1)(2 n-1)}{12 r}-(2 n-1) \chi\left(\mathcal{O}_{X}\right) \\
& \geq \frac{n(n-1)}{4}-\frac{n(n-1)(2 n-1)}{12 \frac{2 n^{2}-3 n+1}{3 n-24 \chi\left(\mathcal{O}_{X}\right)-3}}-(2 n-1) \chi\left(\mathcal{O}_{X}\right) \\
& =2 n \chi\left(\mathcal{O}_{X}\right)-(2 n-1) \chi\left(\mathcal{O}_{X}\right) \\
& =\chi\left(\mathcal{O}_{X}\right) .
\end{aligned}
$$

Hence, $p_{n}=\frac{n(n-1)(2 n-1)}{12} K_{X}^{3}-(2 n-1) \chi\left(\mathcal{O}_{X}\right)+l(n) \geq 2$ since $\chi\left(\mathcal{O}_{X}\right)>0$ and $K_{X}^{3}>0$.

Remark. When $\chi\left(\mathcal{O}_{X}\right)=1, p_{12} \geq 2$ by Theorem 1 if the basket of singularities contains a point of index $r \geq 29$.

Let's modify the second condition in Theorem 1 as follows:

$$
r \geq \max \left\{2 n-2, \frac{1}{3} \frac{n(n-1)(2 n-1)}{n(n-1)-(8 n-4) \chi\left(\mathcal{O}_{X}\right)}\right\} .
$$

Then, in the proof, we have $l(n)-(2 n-1) \chi\left(\mathcal{O}_{X}\right) \geq 0$. Since $K_{X}^{3}>0, p_{n} \geq 1$.

By modified Theorem 1, when $\chi\left(\mathcal{O}_{X}\right)=1, p_{12} \geq 1$ if the basket of singularities contains a point of index $r \geq 26$. Thus, it is enough to consider finite numbers of $r$ to check the nonvanishing of $p_{12}$ since we have an upperbound for $r$. 
Acknowledgement. Author would like to express his gratitude to the referee for kind and constructive suggestions.

\section{References}

[1] A. R. Fletcher, Contributions to Riemann-Roch on projective 3-folds with only canonical singularities and applications, Algebraic geometry, Bowdoin, 1985 (Brunswick, Maine, 1985), 221-231, Proc. Sympos. Pure Math., 46, Part 1, Amer. Math. Soc., Providence, RI, 1987.

[2] M. Reid, Young person's guide to canonical singularities, Algebraic geometry, Bowdoin, 1985 (Brunswick, Maine, 1985), 345-414, Proc. Sympos. Pure Math., 46, Part 1, Amer. Math. Soc., Providence, RI, 1987.

[3] D.-K. Shin, On a computation of plurigenera of a canonical threefold, J. Algebra 309 (2007), no. 2, 559-568.

Department of Mathematics

KONKUK UNIVERSITY

SeOul 143-701, KoreA

E-mail address: dkshin@konkuk.ac.kr 